\title{
The design of remote monitoring system based on virtual serial port Yanqin Sun ${ }^{1, a}{ }^{*}$, Tiancun Yang ${ }^{2, b}$, Heming Cheng ${ }^{3, c}$ \\ ${ }^{1}$ Mechanical and Electrical Engineering, Huanggang Normal College, Huanggang 438000, China asunyanqin923@163.com , bytcgirl@163.com , c184961162@qq.com
}

Keywords: Landslide,Remote monitoring,Data processing,Early warning.

\begin{abstract}
In view of the landslide and slope collapse and other geological disasters phenomenon of instability of rock mass, combined with acoustic emission technology, modern communication and computer technology, and to design the geological disaster monitoring and warning system which has a set of automatic remote measurement, remote data acquisition, data processing, data analysis and forecast function.
\end{abstract}

\section{Introduction}

Today geological disasters often seen in major media, has aroused strong repercussions in the community, relevant government departments and research institutions have organized a large number of experts and scholars to conduct site visits, analysis of the specific reasons for the disaster, and to explore how the timely access to geological previous resulting disaster[1]. The face of widespread and remote monitoring area,we must establish the relevant monitoring mechanism, using a combination of anti-air defense technology to resolve problems such monitoring. In today's era of big things constantly touted, remote monitoring system demand is even more prominent. Therefore, to achieve rock stability index data network security monitoring systems, distance centralized and real-time, which is not only an important direction of the current and future development of a monitoring period.

\section{Remote monitoring system architecture design}

To achieve the intended objectives and requirements, the overall design of the system is guided by the principles of simplicity, flexibility, reliability and economy, through the basic idea of structured systems analysis and design, top-down progressive design, layers of division, ensure each module is relatively independent, reduce or avoid dependency between the data and data redundancy[2,3]. Centralized management requirements of modern equipment intelligent centralized control, the need for on-site real-time data monitoring, storage, diversification function print analysis, mapping, alarms, etc., at the same time, on-site equipment to obey all instructions sent by remote centralized monitoring system, and complete a variety of specified operation at the specified time. Taking into account some of the more complex data, single-chip processing capacity is limited, and therefore the functional requirements of more complex monitoring system, usually a PC to PC, microcontroller as the next crew, both the division of labor, is mainly responsible for a variety of PC complex data processing and control of the microcontroller, the next crew mainly to complete data collection and control devices.

Remote monitoring system uses a multi-tier distributed system architecture, divided into site acquisition layer, data conversion layer, network management layer three levels, with the Internet as the carrier controls the entire process, the use of serial servers to implement remote data transmission. System software architecture using C / S and B / S combination, develop real-time display modules, early warning and forecasting module, data processing module, parameter setting Huanggang Normal University Research Fund project (project number:2015019503) 
module, data landing module, data communication module. Through a unified management platform modules rock landslide monitoring system organically combine to achieve remote remote system control and maintenance. Fig.1 has shown the overall structure of the system .

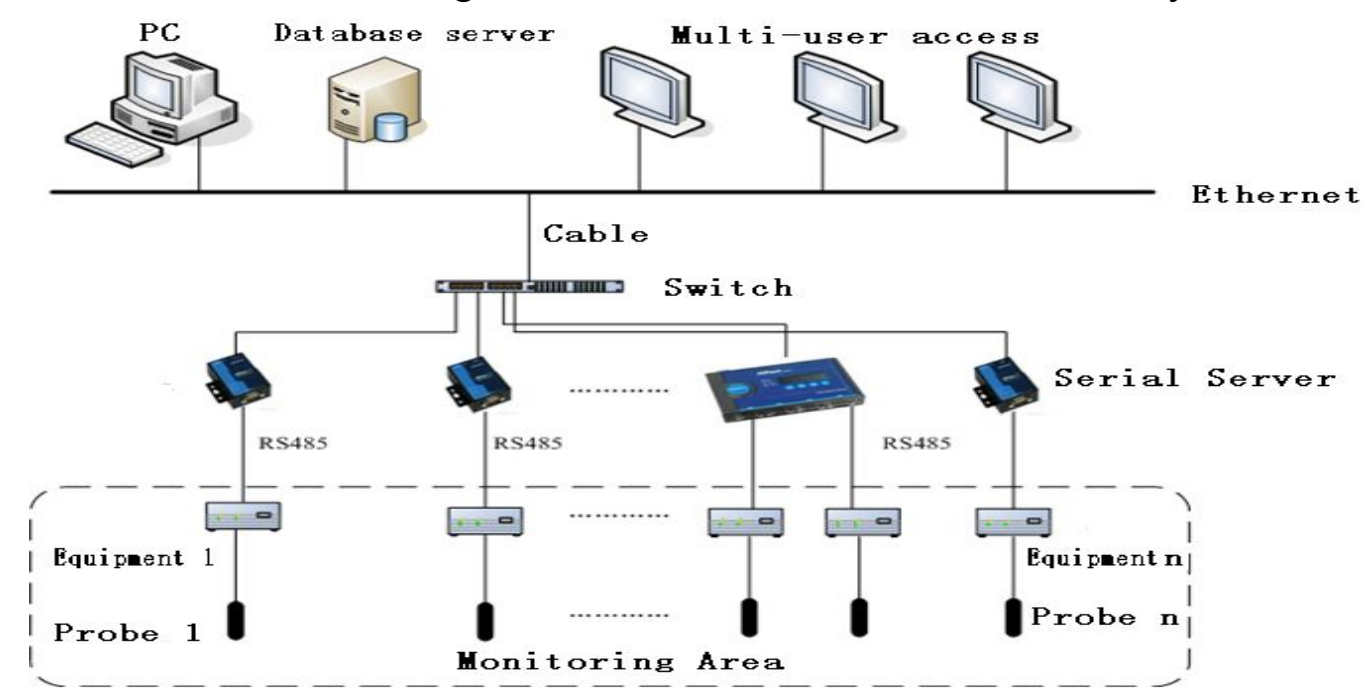

Fig. 1 Acoustic emission remote monitoring system of the whole structure

\section{Communication scheme}

\subsection{Virtual serial port technology}

The system uses a series of MOXA serial server to achieve serial devices networking features to connect to an Ethernet network via this convenient transmission, to meet the needs of remote monitoring[4,5]. Users can be set by software or Web pages NPORT administration mouth networking server parameters, such as the configuration of the serial port parameters: baud rate, data bits, stop bits, parity and so on; the configuration mode for the Real Com modes, upper and lower computer via a communication protocol, serial communications. The serial port server operating mode is set to Real com, namely real serial mode, so everything looks exactly the same as before the serial port, do not need to re-add circuits or develop the network interface, the serial communication is encapsulated in the network in order to achieve the lowest cost virtual Serial remote Ethernet-based communication.

Fig. 2 has based communication program shown in virtual serial port technology. Serial Server PC client software that comes with a virtual COM3, and a serial port server binding, then the user program can read and write to achieve COM3 serial networking and communications modules.

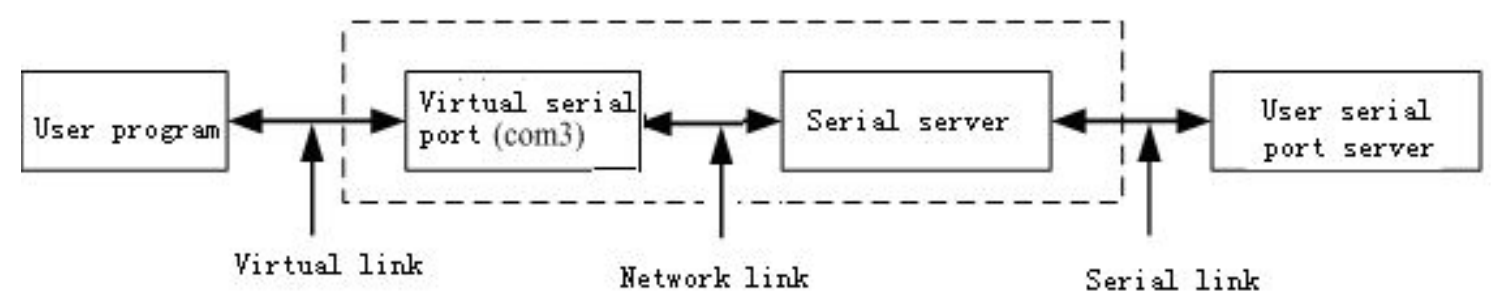

Fig .2 Based on technology of virtual serial communication program

\subsection{Serial communication protocol}

The system uses RS485 serial communication protocol : the transmission rate $9600 \mathrm{bps}, 1$ start bit, 8 data bits, 1 stop bit, no parity. Transfer the contents of the packet, in bytes, the first to send 
low, high evacuation; data format is hexadecimal; asynchronous communication, using the start and stop bits for judging communication. Command information includes commands, data or status information. The usual format: function word + parameters $+\ldots$, where the functional information in the first word of a single byte, used to illustrate this frame required to complete the function. According to different functions, brought back different parameters. Provide nine kinds of commands species in this system, respectively, to achieve different functions. Transmission data is divided into two upstream and downstream directions for multipoint asynchronous two-way data transfer and synchronize one-way broadcast data transfer. Among them, the following line or send data refers to the primary site data transfer to a multi-point or single point; uplink refers downside target of the command received in response to the down command to make a response, synchronous command without response. The following command in the main details of the case does not need to be forwarded, synchronous beat, the status inquiry, the data read, data deletion and synchronous rhythm stop command format. Table 1 has shown the meaning of Functions words.

Table 1 Function word description

\begin{tabular}{|l|l|l|l|}
\hline Word & Function & Word & Function \\
\hline $0 \mathrm{x} 01$ & Status & $0 \mathrm{x} 06$ & Data Delete Status \\
\hline $0 \mathrm{x} 02$ & working mode & $0 \mathrm{x} 07$ & sync beats stop sequence \\
\hline $0 \mathrm{x} 03$ & read data & $0 \mathrm{x} 08$ & environmental parameters read \\
\hline $0 \mathrm{x} 04$ & Synchronize the beat generation sequence & $0 \mathrm{x} 09$ & serial number data deleted \\
\hline $0 \mathrm{x} 05$ & parameters query & & \\
\hline
\end{tabular}

\section{Conclusion}

This article is for multi-channel data acquisition, in order to verify the reliability of the program, on-site laboratory simulation of acoustic emission data collected six channels. Before the system begins operation, to ensure that all equipment wiring is correct, the power supply is normal, set the serial port server the correct IP address, ensure uniqueness and PC in the same segment, as well as virtual serial number. The experiment proved that each of the data transmission channels operate independently without disturbing each other, after the serial data is received immediately forward this data to the appropriate TCP / IP connection, without any wait and intermediate buffering, high speed, high efficiency, and provide a simple the man-machine interface.

\section{References}

[1] Laurence J. Jacobs, William R. Scott, Dianne M. Granata.Experimental and analytical characterization of acoustic emission signals[J].Journal of Nondestructive Evaluation, 1991, 10(2):208 211.

[2] Chang Zhou Romania, Chen Jiangchuan .RAEM rock acoustic emission monitoring system [J]. Metal Materials and Metallurgy Engineering,2012,40(10):135 137.

[3] Tor Erling Unander.Analysis Of Acoustic Emission Waveforms In Rock[J].Research in Nondestructive Evaluation, 2004,15(3):119 148. 
[4] XU Lei-lu,YU Zi-rong.Communication Between SR23 and PC Based on SerialPort in C\#.NET[J].Computer and Modernization, 2011(5):107 109.

[5] Yan Bao zhong. Serial server data acquisition system application [J]. Applied Science and Technology,2008, 35 (12): 40 42. 